\title{
Psychosocial status during the prevalence of COVID-19 disease: the comparison between healthcare workers and general population
}

\author{
Mohammad Ali Zakeri ${ }^{1}$ (D) Sayed Mortaza Hossini Rafsanjanipoor ${ }^{2}$ (D) Nadia Sedri $^{3}$ (D) Mahmood Kahnooji $^{4}$ (D)

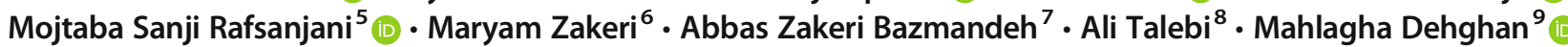

Accepted: 5 March 2021 / Published online: 16 March 2021

(C) The Author(s), under exclusive licence to Springer Science+Business Media, LLC, part of Springer Nature 2021

\begin{abstract}
The prevalence of COVID-19 disease continues to be a significant psychosocial status among health care workers (HCWs) and the general population worldwide. This cross sectional study aimed to compare the psychosocial status between healthcare workers and general population during the prevalence of COVID-19 disease in southeast Iran. Totally 415 health care workers of a medical service center for COVID-19 patients and 1023 people of general population participated in the study. An online socio-demographic characteristics questionnaire, General Health Questionnaire (GHQ -28), and Generalized Anxiety Disorder 7-item (GAD-7) were utilized to evaluate psychosocial status. According to GHQ, the psychological disorders of the HCWs were significantly higher than that of the general population $(P<0.001)$. According to GAD-7, no significant difference was found between general population and HCWs. Multivariate logistic analysis showed no difference between general population and HCWs in the psychological disorder. Although HCWs suffered from psychological disorders more than general population, nearly one third to half of the participants in both groups had psychosocial disorders.
\end{abstract}

Keywords COVID-19 $\cdot$ Health care workers $\cdot$ General population $\cdot$ General anxiety disorders $\cdot$ Psychosocial status

Mahlagha Dehghan

m_dehghan86@yahoo.com; m_dehghan@kmu.ac.ir

Mohammad Ali Zakeri

ma.zakeri115@gmail.com; mzakeri@ rums.ac.ir

Sayed Mortaza Hossini Rafsanjanipoor

mhosseini622@gmail.com

Nadia Sedri

Nadia.sa18@yahoo.com

Mahmood Kahnooji

Drmahmodkahnoji@gmail.com

Mojtaba Sanji Rafsanjani

dr.msanji@gmail.com

Maryam Zakeri

mzakeri36@yahoo.com

Abbas Zakeri Bazmandeh

azaker96@gmail.com

Ali Talebi

at3051756016@gmail.com
1 Determinants of Health Research Centre, Non-Communicable Diseases Research Center, Rafsanjan University of Medical Sciences, Rafsanjan, Iran

2 Social Determinants of Health Research Centre, Rafsanjan University of Medical Sciences, Rafsanjan, Iran

3 Student Research Committee, Zarand faculty of Nursing, Kerman University of Medical Sciences, Kerman, Iran

4 Department of internal medicine, Faculty of medicine, Rafsanjan University of Medical Sciences, Rafsanjan, Iran

5 Emergency Department, Ali Ebn Abitaleb Hospital, Faculty of Medicine, Rafsanjan University of Medical Sciences, Rafsanjan, Iran

6 Physiology-Pharmacology Research Center, Rafsanjan University of Medical Sciences, Rafsanjan, Iran

7 Department of Medical Nanotechnology, School of Advanced Medical Sciences and Technologies, Shiraz University of Medical Sciences, Shiraz, Iran

8 Student Research Committee, School of Nursing and Midwifery, Rafsanjan University of Medical Sciences, Rafsanjan, Iran

9 Nursing Research Center, Kerman University of Medical Sciences, Kerman, Iran 


\section{Introduction}

COVID-19 disease, found in Wuhan, China at the end of 2019, has widely spread worldwide (Bo et al., 2020). One of the main challenges to global public health in the twenty-first century is infectious diseases (Mak et al., 2010). These diseases all affect health physically and psychologically (Xiang et al., 2014). Psychological symptoms such as stress, anxiety, and depression are at high levels in a person infected with COVID-19, who has clinical symptoms of shivers, fevers, nausea, dizziness, muscle weakness and sore throat, poor health status and incidence of chronic disease(Wang et al., 2020).

According to the World Health Organization (WHO) on April 27, 2020, 109,577 laboratory-confirmed COVID-19 incidents, which include 3809 deaths, were widely reported in Hubei province of China (67,743 incidents, 3007 deaths), and 28,673 incidents of 104 other countries (Zhu et al., 2020). In addition, by the increasing number of deaths and cases,COVID-19 has global individual, social, and psychosocial consequences (Bo et al., 2020) in addition to physical problems (Natasha et al., 2020). The COVID-19 virus has caused public panic and psychological health distress (Hossini Rafsanjanipoor et al., 2021).

A wide news reports of the epidemic would still influence the public psychosocial behavior against infectious disease (Rodin et al., 2019). Threat will definitely raise fear while acting as a main motivation to promote preventative behavior (Tang et al., 2018). Xiang et al. concluded that patients suspected of the infection, family members who were quarantined and medical staff need mental health (Xiang et al., 2020). Important psychological morbidities ranged from symptoms and signs of post-traumatic stress disorder such as depression, anxiety, distress and psychosocial attacks to delirium and psychosis (Müller, 2014). The COVID-19 epidemic may initiate new psychotic disorders in the general population without mental disorder at the individual level, also irritates others to mental issues disorder and triggers anxiety to the medical staff of the individuals impacted. Irrespective of contact, people may experience anxiety and fear about being sick or dying, powerless or blaming those who are sick, causing severe a mental breakdown (Hall et al., 2008). Increasing incidence of psychological distress with longer quarantine duration is related to a higher prevalence of depressive symptoms (Hawryluck et al., 2004).

Some studies have shown the association between inadequate coping mechanisms and depression to equate the use of proactive or harmful coping mechanisms with psychological disorders such as depression (Guardino \& Dunkel Schetter, 2014). All feelings can be influenced by depression and anxiety, and this can greatly affect people's behavior and social interaction to others (Ho et al., 2020). Since after death the infection can be spread, traditional burial procedures are not performed such disturbances to traditional processes can lead to anger or anxiety, which can limit access to support from the community (Organization, 2014). Even a sense of sadness is felt while hearing about the deaths of community members in general. When communities face changing positions to respond to the care givers, teachers, and community members, this can have more psychosocial outcomes (O'Carroll, 2015).

Authors believe that quarantine has led to social isolation(Cheung, 2015). In addition, with the closing of public, economic and educational services, decreased health and social facilities, and disruptions in business and industry, most people faced unemployment, which worsened their feelings. The epidemic seriously damaged health services in the affected countries and reduced significantly their capacities (Van Bortel et al., 2016).

HCWs are at high risk of psychological morbidity and should be identified for early psychologically targeted interventions (Ho et al., 2020). Clinical respondents, including emergency staff and HCWs, can also experience elevated anxiety, be physically and mentally depressed and have higher levels of anxiety and worry (Zakeri et al., 2021). The HCWs' fear and anxiety is more due to their being at increased risk of the infection. Perhaps there is a concern that it would spread to their family members and kids. To several HCWs, the work tasks and responsibilities, humanism and personal anxiety often create tension and stress (Tiong \& Koh, 2013). Psychosocial risk factors are not limited to work-related stress (Chirico, 2015), rather they include new and emerging psychosocial risk factors, such as the combined exposure to physical and psychosocial risks, job insecurity, work intensification and high demands at work, high emotional load related to burnout, work-life balance problems, and violence and harassment at work (F Chirico, 2017; Francesco Chirico, 2017a, 2017b). Occupational health surveillance and workplace health promotion are crucial to address psychological risks in general and the pandemic particularly (Chirico, 2016; Chirico et al., 2020).

Most studies suggest that the psychological and social condition has significant effect on the lifestyle of all the people during the prevalence of COVID-19 disease. To date, few studies (Krishnamoorthy et al., 2020) have been published on the pattern of psychosocial status, the comparison between healthcare workers and general population during the prevalence of COVID-19 disease. There has also been insufficient research to investigate the psychological impact of the disease in HCWs (Ho et al., 2020). Therefore, the present study was conducted with the following specific objectives during the outbreak of COVID-19. (a) General health status of participants (General population and HCWs) based on the GHQ -28 ; (b) generalized anxiety disorder of participants (General population and HCWs) based on the GAD-7; (c) Correlation of participants' demographic characteristics with the psychosocial disorder (separately in general population 
and HCWs); (d) Comparison of the general health and generalized anxiety disorder between the general population and $\mathrm{HCWs}$; and (e) assessment of the association of all important study variables with psychological disorders (Yes/No) using multivariate logistic regression.

\section{Methods}

\section{Study Design and Setting}

This cross-sectional analysis investigated the psychosocial status of Rafsanjani citizens and HCWs from April 20 to May 302,020.

\section{Sample Size and Sampling}

Convenience sampling was used for data collection. All citizens living in Rafsanjan city and villages were eligible to participate in the study. Rafsanjan, located in the southwest of Iran has a population of 302,789 people. The city has two government hospitals where only one of them is the referral hospital for COVID-19 patients. The research samples included 630 frontline health care providers caring for COVID-19 patients. Inclusion criteria included participants aged over 18 years with no history of mental disorders (Self-reported). The exclusion criterion was incomplete questionnaires (More than $10 \%$ missing values).

Out of $630 \mathrm{HCWs}, 415$ ones completed the questionnaires, and no questionnaire was deleted. Therefore, the effective response rate of the HCWs was $65.87 \%$. Out of 1087 general population, 1023 completed the questionnaires, 60 of whom were excluded from the study because of high missing value, and 4 were excluded due to the history of mental disorders. Therefore, the effective response rate of the general population was $94.11 \%$. Finally, the data of 1438 participants were used in the final analysis.

\section{Measurements}

Data were collected from a three-part questionnaire for HCWs and general population: socio-demographic characteristics, General Health Questionnaire (GHQ -28), and Generalized Anxiety Disorder 7-item (GAD-7).

Socio-Demographic Characteristics they include gender, age, marital status, level of education, income, and some questions about the coronavirus outbreak, including if anyone in the participant's relatives/ friends has been infected with the coronavirus, if the participant him/herself is at risk for the coronavirus, and the most important concern about the coronavirus.
General Health Questionnaire (GHQ -28) GHQ-28 was used by Goldberg (1972) to assess people's general health and diagnose mental disorders last month (Goldberg, 1972). The GHQ-28 subscale includes somatic symptoms (7 items), anxiety and insomnia (7 items), social impairment ( 7 items) and severe depression (7 items). Each item is rated from 0 to 3 . The total GHQ score is from 0 to 84 , with higher scores indicating more mental disorder (scores $\geq 23$ indicate people with psychological disorder) (Rahmani et al., 2010). In Rahmani et al.'s study, the validity of the questionnaire was 0.90 using a correlation coefficient and the reliability was 0.85 using Cronbach's alpha (Rahmani et al., 2010). In the current analysis, the alpha Cronbach for GHQ was 0.93.

Generalized Anxiety Disorder 7-Item (GAD-7) Spitzer et al. developed the GAD-7 to measure worry and anxiety symptoms. The instrument has seven main items and one additional question to measure the degree of anxiety interference with a person's functioning. The questions are graded from 0 to 3 . Therefore, the total scores of GAD range from 0 to 21 with higher scores reflecting greater anxiety. Scores $\geq 10$ are considered to be in the clinical range i.e. the positive generalized anxiety disorder(Spitzer et al., 2006). The GAD-7 has shown good reliability and validity (Kroenke et al., 2007; Löwe et al., 2008). In Iran, the validity and reliability of this instrument were confirmed and the Cronbach's alpha for the GAD-7 scale was 0.74(Naeinian et al., 2011). In the present research, the Cronbach alpha for the GAD-7 scale was 0.88 .

\section{Data Collection and Data Analysis}

The researcher began sampling after obtaining the necessary permits. Participants were frontline HCWs from one hospital in Rafsanjan $(n=415)$ and general population in Rafsanjan ( $n=$ 1023). We collected data of the HCWs and general population from April 20 to May 302,020. For general population sampling, one of the researchers distributed an online questionnaire on social networks (WhatsApp, Telegram, Eita, Soroush and I-Gap). For HCWs, the researcher began sampling after obtaining the necessary permits. The researcher referred to the hospital during different shifts and began sampling when HCWs had the opportunity to complete the questionnaire. One trained researcher, who was familiar with the objectives of the study, distributed the questionnaire to collect information faster and on a specific schedule.

SPSS 25 has been used for analysis of the data. Frequency, percentage, mean and standard deviation were used to define the characteristics of the study, the GHQ and GAD-7 levels. The chi square test was used to confirm the correlation between qualitative variables and psychological disorder (yes/no). Multivariate logistic regression was used to evaluate the relation between variables of analysis and psychological disorder. The missing values of the GHQ and GAD-7 questionnaires were replaced with median. The level of significance was considered 0.05 . 


\section{Ethical Considerations}

The study protocol was approved by Rafsanjan University of Medical Sciences (IR.RUMS.REC.1399.004 March 27, 2020, and IR.RUMS.REC.1399.017 April 8, 2020,). The first page of online questionnaire explained some information about the study objectives, confidentiality and anonymity of the information, and the voluntary participation.

\section{Results}

The participants' characteristics are presented in Table 1 . According to GHQ, 29.8\% ( $n=306)$ of the general population and $48.2 \%(n=200)$ of the HCWs had psychological disorders. There were significant differences between general population and HCWs in general health $(p<0.001)$ and all its dimensions, including somatic symptoms, anxiety and insomnia, social impairment $(\mathrm{p}<0.001)$, and severe depression $(p=$ 0.01 ). On the other hand, the psychological disorders were significantly higher in HCWs compared with the general population (Table 2). According to GAD-7, 28.5\% $(n=292)$ of the general population and $27 \%(n=112)$ of the HCWs had generalized anxiety disorders. No significant difference was found between general population and HCWs in GAD ( $p=$ 0.99) (Table 2).

In general population, the bivariate analysis showed a significant association between psychosocial disorder, gender $(\mathrm{p}<$ $0.001)$, age $(\mathrm{p}<0.001)$, marital status $(p=0.007)$, income $(\mathrm{p}<$ $0.001)$, thinking of being infected with the coronavirus $(\mathrm{p}<$ 0.001 ), and the most important concern about Coronavirus $(p=0.001)$ (Table 1). In HCWs, the bivariate analysis showed a significant association between psychosocial disorder, gender $(p=0.04)$ and income $(\mathrm{p}=0.01)($ Table 1$)$.

The multivariate logistic regression with backward method was conducted for further analysis. The results showed that only gender $(\mathrm{p}<0.001)$, marital status $(\mathrm{p}=0.01)$, income $(\mathrm{p}<$ $0.001)$, being at risk for infection with the coronavirus $(\mathrm{p}<$ 0.001 ), and the most important concern about coronavirus $(p=0.03)$ were significantly associated with psychological disorders in the total samples. On the other hand, the risk of psychosocial disorder was higher in females than male (OR: 1.69, 95\%CI: 1.32-2.16). In addition, the risk of psychosocial disorder was higher in the unmarried than married, those earning income less than 1 million than those earning higher income, and those whose most important concern about coronavirus was family getting sick and death. The risk of psychosocial disorder was also higher in those who thought that they were at risk of the coronavirus than others (OR: $1.66,95 \% \mathrm{CI}$ : 1.31-2.12). Multivariate logistic analysis showed no difference between general population and HCWs in the psychological disorder (Table 3).

\section{Discussion}

The findings of the current study revealed significant differences between the general population and HCWs in general health and all its dimensions with two-variable analysis. On the other hand, the psychological disorders were significantly higher in the HCWs compared with general population $(P<0.001)$.

Psychological disorders are important in the prevalence of COVID-19 disease and many studies tackled this issue. Wang et al. indicated that $53.8 \%$ of the participants had mild to extreme psychological disorders during the COVID-19 epidemic (Wang et al., 2020), which was in line with the findings of the present study. Results of the present study have shown higher psychological disorders in the HCWs. Naushad et al. studied the impact of disasters on the HCW's mental health and concluded that the main risk factors reported for the development of psychological disorders included a loss of community assistance and interaction, behavioral problems and lack of education (Naushad et al., 2019). Lu et al. found that $17.3 \%$ of the HCWs had mental symptoms during the SARS epidemic (Lu et al., 2006).

One of the psychiatric problems facing staff was that they did not know how to quarantine patients in the hospital. In addition, the HCWs are concerned about the lack of protective equipment and feelings of disability when facing sick people. Most research conducted on interventional methods to enhance mental health among the HCWs have demonstrated the relationship between good mental health and management of viral infections, including COVID 19 disease. Xiang et al. reported that psychotherapy in COVID-19 disease was urgently needed. They showed psychological disorders in general population and HCWs, and the results were consistent with the present study findings (Xiang et al., 2020).

Maintaining mental health services for $\mathrm{HCW}$ is important to help manage infectious diseases (Chen et al., 2020). According to GAD-7, 28.5\% of the general population and $27 \%$ of the HCWs had generalized anxiety disorders. No significant difference was found between general population and HCWs in GAD. Zhu et al. found that the Chinese suffered from extreme anxiety because of no masks and protective equipment on the market (Zhu et al., 2020). Their results confirm the results of the present study regarding anxiety disorders. Psychological fear and generalized anxiety disorders may be more intense with increased global loneliness due to lockdown (Tang et al., 2018).

Wang et al. indicated that $28.8 \%$ of the participants had mild to extreme signs of anxiety, $8.1 \%$ had moderate to serious stress and $16.5 \%$ had mild to serious symptoms of depression (Wang et al., 2020), which is consistent with this study in general health factors such as, anxiety and severe depression.

The findings of this research showed an equal level of generalized anxiety disorders in both groups, and no 


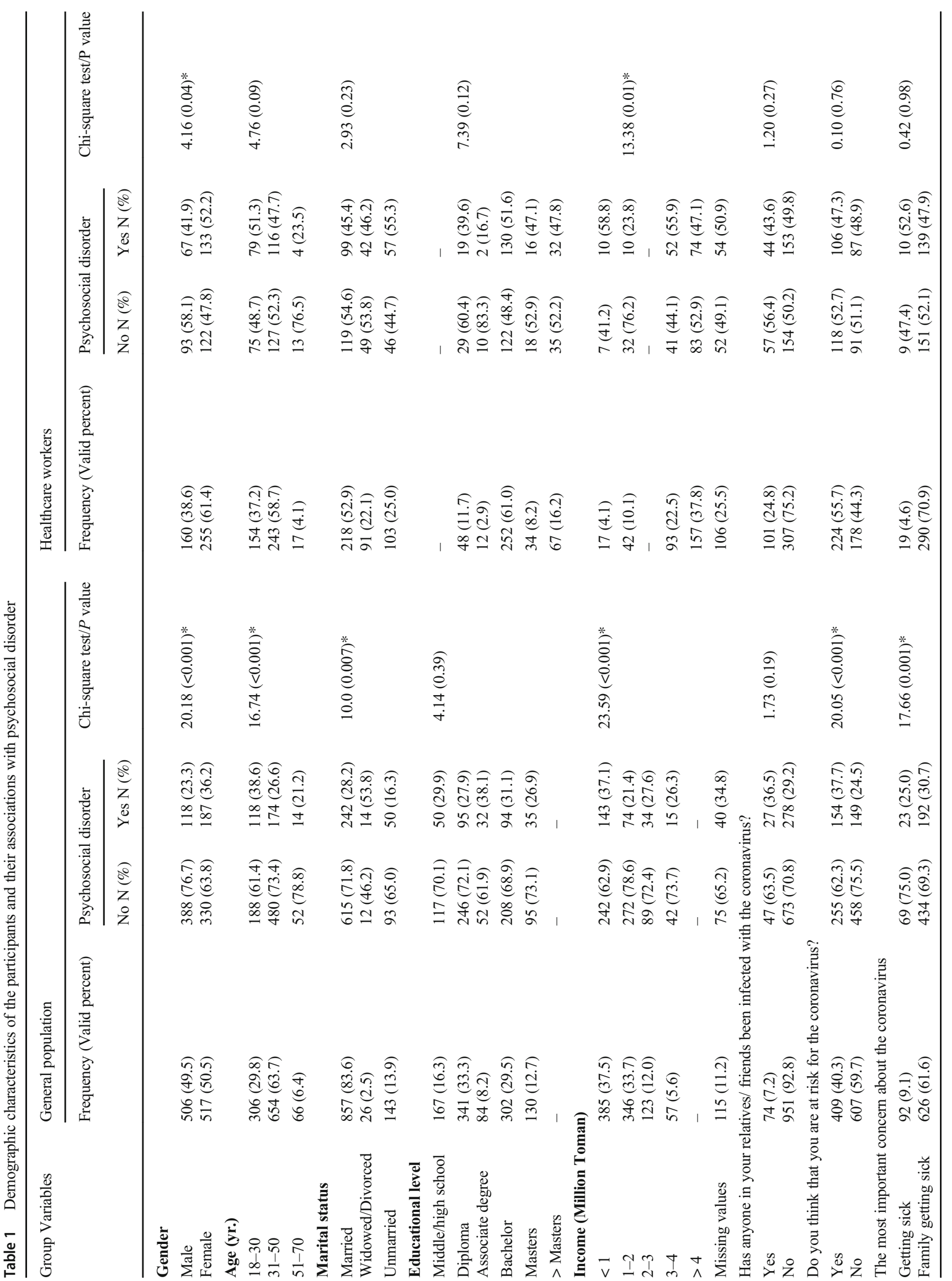


significant difference was found between the two groups. However, generalized anxiety disorders are present during the prevalence of COVID-19 disease and can interrupt people's and health care workers' daily life processes and also cause some disorders such as insomnia, depression, social impairment and even embitter physical symptoms, so anxiety disorders can be an important issue (Hossini Rafsanjanipoor et al., 2021; Zakeri et al., 2021).

The bivariate analysis in this study demonstrated the relationship between psychosocial disorder and gender in both general population and HCWs, and the multivariate logistic regression showed the higher risk of psychosocial disorder in females. Ho et al. conducted a research on mental health strategies to combat the psychological impact of COVID-19 beyond paranoia and panic, and suggested that the prevalence of fear resulting in psychological behavior among people in the early stages of infectious outbreaks was a phenomenon that was obviously not unusual as people of a particular gender can be infected (Ho et al., 2020). This study is congruent with the present study in the gender variable. This research clearly showed a significant difference between men and women in psychosocial disorders, and the number of females in general population and HCWs was greater than that of men, while in the study of Ho et al. (Ho et al., 2020), psychological fear was not mentioned in any particular gender.

According to Wang et al. research in general population, females have reported experiencing an increased stress, level of psychological influence of outbreak, mental health problems and fear (Wang et al., 2020), which is consistent with this study and that could possibly be due to their unique socioeconomic and biological conditions and also an elevated risk of depression in women (Lim et al., 2018). Females are experiencing greater negative perceptions or mood changes. Hormone levels in ovaries are responsible for impaired response to stimulate emotion in some cycles from the menstruation process that may form a particular psychological vulnerability in women(Soni et al., 2013). Yang et al. showed that female participants experienced the most adverse effects in symptoms of cognitive function or feeling than men (Yang et al., 2019). Experimental research showed that in nervous systems related to worry and excitement, women were more reactive than men (Felmingham et al., 2010). All these results confirm the present study regarding higher prevalence of the symptoms in females.

Multivariate logistic regression analysis revealed that the risk of psychosocial disorder was higher in those earning income less than 1 million. Furthermore, the bivariate analysis showed the relationship between psychosocial disorder and income in both groups. Liang et al. suggested that the PTSD levels of traders were comparatively higher than those of other working classes (Liang et al., 2020). When COVID-19 occurred, the work culture of all people changed. They would develop higher levels of depression, since they cannot adjust 
Table 2 Comparison of the general health and generalized anxiety disorder between general population and healthcare workers

\begin{tabular}{|c|c|c|c|c|c|c|c|}
\hline \multirow[t]{2}{*}{ Group Variables } & \multicolumn{2}{|c|}{ General population } & \multicolumn{2}{|c|}{ Healthcare workers } & \multirow[t]{2}{*}{ Independent $t$ test } & \multirow[t]{2}{*}{ Effect size } & \multirow[t]{2}{*}{$P$ value } \\
\hline & Mean & Standard Deviation & Mean & Standard Deviation & & & \\
\hline General health & 19.78 & 10.79 & 23.89 & 12.68 & 6.22 & 0.35 & $<0.001$ \\
\hline Somatic symptoms & 5.16 & 3.32 & 6.67 & 4.07 & 7.33 & 0.41 & $<0.001$ \\
\hline Anxiety and insomnia & 5.84 & 3.26 & 6.73 & 4.62 & 4.15 & 0.22 & $<0.001$ \\
\hline Social impairment & 6.27 & 3.30 & 7.49 & 3.24 & 6.38 & 0.37 & $<0.001$ \\
\hline Severe depression & 2.51 & 3.15 & 2.99 & 3.81 & 2.47 & 0.14 & 0.01 \\
\hline Generalized Anxiety Disorder & 7.01 & 5.32 & 7.01 & 5.11 & -0.02 & 0.0 & 0.99 \\
\hline
\end{tabular}

to these rapid variations in work culture in a short period. Loss of job during quarantine or job changes that earned less than before the COVID-19 period were important issue of psychosocial disorders for the people.

The bivariate analysis in general population and the multivariate logistic regression in total samples revealed that the

Table 3 The logistic model of associations of important variables with general health

\begin{tabular}{lccc}
\hline Variable & \multicolumn{2}{l}{ Multivariate logistic regression } \\
\cline { 2 - 4 } & $\mathrm{B}$ & $95 \%$ Confidence interval for B & P value \\
\hline Gender & & & \\
Male & 1 & & $<0.001$ \\
Female & 1.69 & $1.32-2.16$ & \\
Marital status & & & 0.01 \\
Married & 1 & & 0.06 \\
Widowed/Divorced & 1.38 & $0.99-1.92$ & 0.008 \\
Unmarried & 1.84 & $1.17-2.87$ & \\
Income (Million Riyal) & & $<0.001$ \\
$<1$ & 1 & & $<0.001$ \\
1-2 & 0.49 & $0.35-0.69$ & 0.38 \\
$2-3$ & 0.81 & $0.50-1.30$ & 0.19 \\
$3-4$ & 1.32 & $1.32-0.87$ & 0.37 \\
$>4$ & 1.20 & $0.80-1.81$ & 0.95 \\
Unknown & 1.01 & $0.69-1.48$ &
\end{tabular}

Do you think you are at risk for infection with the coronavirus?

\begin{tabular}{llll} 
No & 1 & & \\
Yes & 1.66 & $1.31-2.12$ & $<0.001$ \\
What is your most important concern about Coronavirus? & \\
No concern & 1 & & 0.03 \\
Getting sick & 1.75 & $0.91-3.38$ & 0.09 \\
Family getting sick & 2.06 & $1.23-3.43$ & 0.006 \\
Death & 2.52 & $1.43-4.43$ & 0.001 \\
Others & 1.89 & $0.87-4.09$ & 0.11 \\
\hline
\end{tabular}

$1=$ the reference unmarried participants had significantly higher psychosocial disorders; this result was consistent with that of Liang et al. (Liang et al., 2020). This difference may be due to family relationship and the feeling of loneliness and hopeless in single participants. Lack of support for negative feelings or signs of depression could be a good reason to enhance psychosocial disorder in unmarried people. Qu et al. have demonstrated that unmarried people had no opportunity to receive family support for coping to psychiatric problems such as anxiety and depression, underlining the value of helping family throughout this emergency (Qu et al., 2014).

The multivariate logistic regression showed that the risk of psychosocial disorder was higher in those thinking of being at risk for infection with the coronavirus and those whose most important concern about coronavirus was family getting sick and death. In general population, the bivariate analysis showed a significant association between psychosocial disorder and thinking of being at risk for infection with the COVID-19. In total samples, these issues may be due to the uncertainty about the disease, such as when COVID-19 is controlled or how it can be managed. New medicines for the potential treatment, their efficacies and side effects are a significant issue and can be a cause of concern to everyone. Most importantly, how to care for an infected person, whether it is mild or moderate or severe, and whether or not it can be treated, and if the people contacting him are infected or not. Thinking of being at risk for infection with the coronavirus is one of the psychological disorders in HCWs that may be improved with transfer of the hospital instructions for COVID19 to all staff and following clear procedures in infection management is essential. There must also be protective measures to ensure that HCWs will not become infected when exposing to the COVID-19 at the workplace. In addition, a support network should be developed to assist HCWs in times of need at work. It is important to identify mentally depressed individuals, allocate prompt support to them and personnel can move on without worry of blaming (Ho et al., 2020).

General papulation think that they are at risk for infection with the COVID19 because of lack of awareness about the 
accurate health information such as associated risk factors, the route of exposure, response to therapy and the mode of transmission. If HCWs get accurate evidence and trust on the health policy and strategies for COVID19 control, it may significantly decrease worry and encourage to properly implement the preventive strategies (Ho et al., 2020). Chen et al. reported that nurses displayed aggressiveness, anxiety, refusal to rest and psychological distress. One of the most common causes of mental health issues in HCWs is that they did not want their families to be infected, so they were afraid to carry the virus to their homes (Chen et al., 2020).

\section{Limitations}

This study has several limitations. First, this study was performed on a limited population in one of the provinces of Iran, which makes it difficult to generalize our results to other regions. Second, this study lacks longitudinal follow-up and does not determine the long-term consequences of COVID-19. Therefore, future studies could focus on comparing long-term psychological consequences in these two populations. Third, sampling was done through a social network that could lead to bias. Although the results are for the general population, it is difficult to generalize the results to illiterate people who do not have access to social networks.

\section{Conclusion}

Due to the obvious globalization of the epidemic and the increasing lack of investment in health services, populations are at risk of psychosocial effects that compound health needs. The bivariate analysis in this study showed that psychiatric issues in HCWs was greater than that of the general papulation. On the other hand, the multivariate logistic regression showed that the risk of psychosocial disorder was higher in those thinking of being at risk for infection with the coronavirus, as well as in all samples and those whose most important concern about coronavirus was family getting sick and death. It is acceptable to comprehend the relationship between psychosocial disorder and thinking of being at risk for infection with the coronavirus, especially in the health care workers, because they are the frontline health care providers. This study presents an important insight to the psychosocial issues in general population and HCWs.

Acknowledgements The authors would like to thank the Social Determinants of Health Research Centre for its support and collaboration in Ali Ebn Abitaleb Hospital, Rafsanjan University of Medical Science, Rafsanjan, Iran.

Data Availability Data are available by contacting with the corresponding author by email.

\section{Declarations}

Ethical Approval The code of ethics (IR.RUMS.REC.1399.004 and IR.RUMS.REC.1399.017) was approved by the Ethics Committee of Rafsanjan University of Medical Sciences.

Informed Consent Informed consent was provided in the first page of online questionnaire.

Conflict Interest No conflict of Interest was provided for this paper.

\section{References}

Bo, H.-X., Li, W., Yang, Y., Wang, Y., Zhang, Q., Cheung, T., Wu, X., \& Xiang, Y.-T. (2020). Posttraumatic stress symptoms and attitude toward crisis mental health services among clinically stable patients with COVID-19 in China. Psychological Medicine, 1-7.

Chen, Q., Liang, M., Li, Y., Guo, J., Fei, D., Wang, L., He, L., Sheng, C., Cai, Y., \& Li, X. (2020). Mental health care for medical staff in China during the COVID-19 outbreak. The Lancet Psychiatry, 7(4), e15-e16.

Cheung, E. (2015). An outbreak of fear, rumours and stigma: Psychosocial support for the Ebola virus disease outbreak in West Africa. Intervention, 13(1), 70-76.

Chirico, F. (2015). The assessment of psychosocial risk: Only "workrelated stress" or something else? La Medicina del Lavoro, 106(1), 65-66.

Chirico, F. (2016). Adjustment disorder as an occupational disease: Our experience in Italy. The international journal of occupational and environmental medicine, 7(1), 52-57. https://doi.org/10.15171/ ijoem.12016.15716.

Chirico, F. (2017). Burnout and depression are not the same thing. The British Journal of Psychiatry, eLetter, Oct, 2, (190), 2005-2015 Eletter. Available from: http://bjp.rcpsych.org/content/2190/2001/ 2081.2002.e-letters\#burnout-syndrome-and-depression-are-not-thesame-thing.

Chirico, F. (2017a). The forgotten realm of the new and emerging psychosocial risk factors. Journal of occupational health, 59(5), 433435. https://doi.org/4https://doi.org/10.1539/joh.1517-0111-OP.

Chirico, F. (2017b). Is burnout a syndrome or an occupational disease? Instructions for occupational physicians. Epidemiologia $e$ prevenzione, 41(5-6), 294-298. Doi: 2https://doi.org/10.19191/ EP19117.19195-19196.P19294.19089.

Chirico, F., Nucera, G., \& Magnavita, N. (2020). Protecting the mental health of healthcare workers during the COVID-19 emergency. BJPsych International, 1-2. https://doi.org/10.1192/bji.2020. 1139 [Opens.

Felmingham, K., Williams, L. M., Kemp, A. H., Liddell, B., Falconer, E., Peduto, A., \& Bryant, R. (2010). Neural responses to masked fear faces: Sex differences and trauma exposure in posttraumatic stress disorder. Journal of Abnormal Psychology, 119(1), 241-247.

Goldberg, D. (1972). The detection of psychiatric illness by questionnaire: A technique for the identification and assessment of nonpsychotic psychiatric illness. London, England: Oxford University Press.

Guardino, C. M., \& Dunkel Schetter, C. (2014). Coping during pregnancy: A systematic review and recommendations. Health Psychology Review, 8(1), 70-94.

Hall, R. C., Hall, R. C., \& Chapman, M. J. (2008). The 1995 Kikwit Ebola outbreak: Lessons hospitals and physicians can apply to future viral epidemics. General Hospital Psychiatry, 30(5), 446-452.

Hawryluck, L., Gold, W. L., Robinson, S., Pogorski, S., Galea, S., \& Styra, R. (2004). SARS control and psychological effects of 
quarantine, Toronto, Canada. Emerging Infectious Diseases, 10(7), 1206-1212.

Ho, C. S., Chee, C. Y., \& Ho, R. C. (2020). Mental health strategies to combat the psychological impact of COVID-19 beyond paranoia and panic. Annals of the Academy of Medicine, Singapore, 49(1), 1-3.

Hossini Rafsanjanipoor, S. M., Zakeri, M. A., Dehghan, M., Kahnooji, M., Sanji Rafsanjani, M., Ahmadinia, H., \& Zakeri, M. (2021). Iranian psychosocial status and its determinant factors during the prevalence of COVID-19 disease. Psychology, Health \& Medicine. https://doi.org/10.1080/13548506.13542021.11874438.

Krishnamoorthy, Y., Nagarajan, R., Saya, G. K., \& Menon, V. (2020). Prevalence of psychological morbidities among general population, healthcare workers and COVID-19 patients amidst the COVID-19 pandemic: A systematic review and meta-analysis. Psychiatry Research, 293, 113382.1133https://doi.org/10.111016/ j.psychres.112020.113382.

Kroenke, K., Spitzer, R. L., Williams, J. B. W., Monahan, P. O., \& Lowe, B. (2007). Anxiety disorders in primary care: Prevalence, impairment, comorbidity, and detection. Annals of Internal Medicine, 146(5), 317-325.

Liang, L., Ren, H., Cao, R., Hu, Y., Qin, Z., Li, C., \& Mei, S. (2020). The effect of COVID-19 on youth mental health. Psychiatric Quarterly, 1-12.

Lim, G. Y., Tam, W. W., Lu, Y., Ho, C. S., Zhang, M. W., \& Ho, R. C. (2018). Prevalence of depression in the community from 30 countries between 1994 and 2014. Scientific Reports, 8(1), 1-10.

Löwe, B., Decker, O., Müller, S., Brähler, E., Schellberg, D., Herzog, W., \& Herzberg, P. (2008). Validation and standardization of the generalized anxiety disorder screener (GAD-7) in the general population. Med Care, 46(3), 266-274. Doi: 2https://doi.org/10.1097/MLR. $1090 \mathrm{~b} 1013 \mathrm{e} 318160 \mathrm{~d} 318093$.

Lu, Y.-C., Shu, B.-C., \& Chang, Y.-Y. (2006). The mental health of hospital workers dealing with severe acute respiratory syndrome. Psychotherapy and Psychosomatics, 75(6), 370-375.

Mak, I. W. C., Chu, C. M., Pan, P. C., Yiu, M. G. C., Ho, S. C., \& Chan, V. L. (2010). Risk factors for chronic post-traumatic stress disorder (PTSD) in SARS survivors. General Hospital Psychiatry, 32(6), $590-598$.

Müller, N. (2014). Infectious diseases and mental health. Comorbidity of Mental and Physical Disorders, 99.

Naeinian, M., Shaeiri, M., Sharif, M., \& Hadian, M. (2011). To study reliability and validity for a brief measure for assessing generalized anxiety disorder (GAD-7). Scientific Journal of Clinical Psychology \& Personality, 2(4), 41-50.

Natasha, S., Mansoor, A. D., \& Junaid, R. (2020). Physical and mental health impacts of COVID-19 on healthcare workers: A scoping review. International Journal of Emergency Medicine (Online), 13(1). https://doi.org/10.1186/s12245-12020-00299-12245.

Naushad, V. A., Bierens, J. J., Nishan, K. P., Firjeeth, C. P., Mohammad, O. H., Maliyakkal, A. M., ChaliHadan, S., \& Schreiber, M. D. (2019). A systematic review of the impact of disaster on the mental health of medical responders. Prehospital and Disaster Medicine, 34(6), 632-643.

O'Carroll, L. (2015). Ebola takes mental health toll where 'life has frozen' in Sierra Leone. The Guardian. Available from: https:// www.theguardian.com/global-development/2015/jan/12/ebolamental-health-sierra-leone-depression.

Organization, W. H. (2014). Psychological first aid during Ebola virus disease outbreaks (provisional version). Geneva: WHO.[Google Scholar].

Qu, Z., Wang, C.-W., Zhang, X., Ho, A. H., Wang, X., \& Chan, C. L. (2014). Prevalence and determinants of depression among survivors 8 months after the Wenchuan earthquake. The Journal of Nervous and Mental Disease, 202(4), 275-279.
Rahmani, F., Behshid, M., Zamanzadeh, V., \& Rahmani, F. (2010). Relationship between general health, occupational stress and burnout in critical care nurses of Tabriz teaching hospitals. Iran Journal of Nursing, 23(66), 54-63.

Rodin, P., Ghersetti, M., \& Odén, T. (2019). Disentangling rhetorical subarenas of public health crisis communication: A study of the 2014-2015 Ebola outbreak in the news media and social media in Sweden. Journal of Contingencies and Crisis Management, 27(3), 237-246.

Soni, M., Curran, V. H., \& Kamboj, S. K. (2013). Identification of a narrow post-ovulatory window of vulnerability to distressing involuntary memories in healthy women. Neurobiology of Learning and Memory, 104, 32-38.

Spitzer, R., Kroenke, K., Williams, J., \& Löwe, B. (2006). A brief measure for assessing generalized anxiety disorder: The GAD-7. Archives of Internal Medicine, 166(10), 1092-1097.

Tang, L., Bie, B., Park, S.-E., \& Zhi, D. (2018). Social media and outbreaks of emerging infectious diseases: A systematic review of literature. American Journal of Infection Control, 46(9), 962-972.

Tiong, W. W., \& Koh, G. C. (2013). Ethical considerations in the review of Singapore's H1N1 pandemic response framework in 2009, Annals of the academy of medicine, Singapore., 42(5), 246-250.

Van Bortel, T., Basnayake, A., Wurie, F., Jambai, M., Koroma, A. S., Muana, A. T., Hann, K., Eaton, J., Martin, S., \& Nellums, L. B. (2016). Psychosocial effects of an Ebola outbreak at individual, community and international levels. Bulletin of the World Health Organization, 94(3), 210.

Wang, C., Pan, R., Wan, X., Tan, Y., Xu, L., Ho, C. S., \& Ho, R. C. (2020). Immediate psychological responses and associated factors during the initial stage of the 2019 coronavirus disease (COVID-19) epidemic among the general population in China. International Journal of Environmental Research and Public Health, 17(5), 1729.

Xiang, Y.-T., Yu, X., Ungvari, G. S., Correll, C. U., \& Chiu, H. F.-K. (2014). Outcomes of SARS survivors in China: Not only physical and psychiatric co-morbidities. East Asian Archives of Psychiatry, 24(1), 37.

Xiang, Y.-T., Yang, Y., Li, W., Zhang, L., Zhang, Q., Cheung, T., \& Ng, C. H. (2020). Timely mental health care for the 2019 novel coronavirus outbreak is urgently needed. The Lancet Psychiatry, 7(3), 228229.

Yang, R., Sun, H., Wu, Y., Lu, G., Wang, Y., Li, Q., Zhou, J., Sun, H., \& Sun, L. (2019). Long-lasting sex-specific effects based on emotionand cognition-related behavioral assessment of adult rats after posttraumatic stress disorder from different lengths of maternal separation. Frontiers in Psychiatry, 10, 289.

Zakeri, M. A., Dehghan, M., Ghaedi Heidari, F., Pakdaman, H., Mehdizadeh, M., Ganjah, H., Sanji Rafsanjani, M., Rafsanjanipoor, H., \& S. m. (2021). Mental health outcomes among health care workers during the COVID-19 outbreak in Iran. Mental Health Review Journal. https://doi.org/10.1108/MHRJ-1110-20200075.

Zhu, Z., Xu, S., Wang, H., Liu, Z., Wu, J., Li, G., Miao, J., Zhang, C., Yang, Y., Sun, W., Zhu, S., Fan, Y., Chen, Y., Hu, J., Liu, J., \& Wang W. (2020). COVID-19 in Wuhan: Sociodemographic characteristics and hospital support measures associated with the immediate psychological impact on healthcare workers. EClinical Medicine, 24:100443. https://www.sciencedirect.com/science/ article/pii/S2589537020301875.

Publisher's Note Springer Nature remains neutral with regard to jurisdictional claims in published maps and institutional affiliations. 